\title{
The Emerging Theme of Redox Bioenergetics in Health and Disease
}

\author{
Philip A. Kramer, Victor M. Darley-Usmar
}

\begin{abstract}
Mitochondrial function has long been recognized as central to normal physiology and a contributor to a broad range of pathologies. Much of the early research in mitochondrial biology focused on the mechanisms to generate ATP and characterization of mitochondria from highly energetic tissues such as the heart or liver. More recent studies emphasize the role of mitochondria in redox signaling and in less energetic cells such as those in the innate immune system and the vasculature. In this short overview, we discuss some of these recent developments in translational and basic research in mitochondrial pathophysiology. Advanced high throughput analytical techniques are now allowing the assessment of bioenergetic health in human populations and the emergence of the exciting new field of metabolotherapeutics. These have led to the emergence of the new field of redox bioenergetics which encompasses both the canonical aspects of mitochondrial energy production and the organelles' role in cell signaling and disease.
\end{abstract}

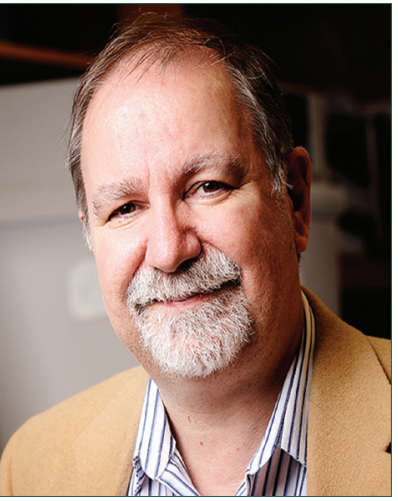

Dr. Victor Darley-Usmar (Biomed J 2015;38:294-300)

\section{Key words: bioenergetic health, biomarker, extracellular flux analysis, metabolism, oxidative stress, signaling}

$\mathrm{M}$ itochondrial biology continues to play a central role in our understanding of the basic biological processes and the pathology of disease. Recent technological advances in cellular respirometry and microscopy have revealed for the first time the dynamic impact of mitochondrial networks, the complexity of mitochondrial metabolism in cells not traditionally viewed as highly energetic, and a completely new pathway for the turnover and removal of "rogue" mitochondria, known as mitophagy. ${ }^{[1-4]}$ Since the discovery of diseases directly attributable to mutations in mitochondrial DNA (mtDNA), the impact of bioenergetic dysfunction has now extended to a broad range of pathologies including diabetes, neurodegeneration, and cardiovascular diseases. ${ }^{[5-11]}$ This, in turn, has ushered onto the stage a broad range of mitochondrial therapeutics which function through different mechanisms, in addition to the recognition that established therapeutics, such as metformin, modulate bioenergetics. ${ }^{[12-17]}$ The role of mitochondria has also developed well beyond simply providing ATP to the cell to encompass a complex retrograde signaling pathway to the nucleus. ${ }^{[18-20]}$ The mechanisms through which this occurs are still not clear, but have been shown in several cases to involve the controlled generation of superoxide and hydrogen peroxide from the respiratory chain. ${ }^{[20-22]}$ Interestingly, among the 13 proteins coded for by the mtDNA are the critical redox centers in the respiratory chain, which offer a mechanism through which mutations in mtDNA could modulate superoxide levels in response to stress and, thus, impact on pathological processes. ${ }^{[23]}$ Taken together, these findings result in the new field of redox bioenergetics.

Our perspective of mitochondrial function is also rapidly changing in response to new findings of cellular bioenergetics in the cells of the innate immune system. ${ }^{[24,25]}$ The early association of cancer cells with an altered bioenergetics metabolism characterized by aerobic glycolysis has now been extended to encompass lymphocytes and monocytes as they adapt to their changing biological functions in normal physiology. ${ }^{[24,26,27]}$ Early studies of mitochondrial function were largely based upon the disruption of tissues such as the heart or liver abundant in the organelle and characterization of the organelles isolated from their cellular milieu. The discovery of a sophisticated molecular postal system which directs proteins to different compartments within the mitochondrion resulted in the widespread notion that the mitochondrial proteome

From the Department of Pathology, UAB Mitochondrial Medicine Laboratory, Center for Free Radical Biology, University of Alabama at Birmingham, Birmingham, AL, USA

Received: Dec. 02, 2014; Accepted: Mar. 29, 2015

Correspondence to: Dr. Victor Darley-Usmar, Department of Pathology, University of Alabama at Birmingham, USA. Biomedical Research

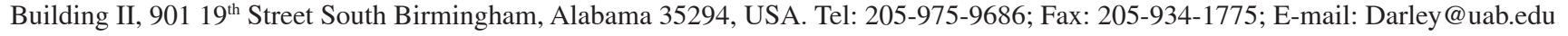

DOI: $10.4103 / 2319-4170.155591$ 
could be discrete and defined for a given cell type and context. However, it is now becoming clear from studies in living cells that a more dynamic interaction of cytosolic proteins with the mitochondrion is occurring over minutes to seconds and is responsive to targeted signaling pathways. For example, functional associations of mitochondria with nitric oxide synthases, NADPH oxidase 4 , and cytochrome $\mathrm{P} 450$ have recently been reported. ${ }^{[28-30]}$ The functional significance of these interactions remains unclear in most cases. In this short overview, we will highlight this theme of redox bioenergetics and the articles supporting these developments from broader literature.

\section{New aspects of mitochondrial function}

The classical approach to measuring mitochondrial function has been to prepare the isolated organelle by disrupting its attachment to the cellular milieu and measuring its activity after purification. It is now technically possible to determine mitochondrial function in a cellular context and then, following permeabilization of the plasma membrane, measure oxidative phosphorylation in the mitochondria from the same cell. ${ }^{[31,32]}$ Interestingly, it is increasingly becoming obvious that the activities of oxidative phosphorylation from isolated mitochondria represent only a small fraction of the metabolic activities they exhibit in the intact cell. ${ }^{[2]}$ In addition, it is now becoming possible to measure cells in atypical circumstances including under low oxygen tension, in spheroids, and to model the air-liquid interface typical of lung epithelial cells. ${ }^{[33-37]}$

The mitochondria are now emerging as a major contributor to a broad range of metabolic diseases and this offers new therapeutic targets both aimed at metabolism and modulating mitochondrial quality control. ${ }^{[2,5,7]}$ Mitochondria are also a target for inflammatory mediators including low levels of $\mathrm{HOCl}$ which can inhibit platelet function. ${ }^{[38]}$ Conversely, mitochondrial toxicity underlies many of the dose-limiting problems with therapeutics and mediates the toxicity of numerous xenobiotics, as will be discussed below. ${ }^{[39]} \mathrm{Sev}-$ eral investigators are developing models for screening for mitochondrial toxicities and one approach has been to substitute galactose for glucose in the media, which forces the cells to rely on mitochondrial function for energetics, thus revealing the bioenergetic defects more readily. ${ }^{[40]}$ These new approaches will bring new insights into how mitochondria behave at the extremes of eukaryotic cellular environments.

\section{Mechanisms of mitochondrial reactive oxygen species and therapeutic targeting}

The mitochondria are both a source and target of reactive oxygen species/reactive nitrogen species (ROS/ RNS), and the interplay between the regulatory processes in which redox signaling molecules modulate the activity of cytoplasmic signaling cascades (so-called retrograde signaling) is emerging as a major area of interest. ${ }^{[19,41-44]}$ The earliest studies relied heavily on mitochondrial inhibitors to identify the potential sites for mitochondrial ROS formation, ${ }^{[45]}$ but, not surprisingly, it has become clear that this is much more complex. In an elegant comprehensive investigation of the sites of ROS formation in skeletal muscle mitochondria, six independent sites were identified which are differentiated by their relative activity and substrate dependency. ${ }^{[46]}$ How the signals from mitochondrial ROS are transduced to modify cell signaling pathways remains an active area of research. Interestingly, it is now becoming clear that the mitochondrial genetic background is capable of conferring resistance or suceptability to cardiovascular disease by modulating redox signaling. ${ }^{[23]}$ One concept that is gaining acceptance is that it is the modulation of the mitochondrial protein thiol networks which are an essential intermediate in retrograde signaling pathways. ${ }^{[41]}$ Interestingly, except in some specialized cases, protein thiols are not very reactive with hydrogen peroxide. This has led to the suggestion that localized and controlled formation of hydrogen peroxide in the respiratory chain can lead to lipid peroxidation. The resulting reactive lipid species can act as second messengers for cell signaling by modifying protein thiols. ${ }^{[22,47]}$ This opens up the possibility, which is gaining experimental evidence, that electrophile therapeutics and natural products, such as curcumin, are capable of modulating the mitochondrial function through electrophilic signaling. ${ }^{[48,49]}$ An interesting development in this area is the idea that the reaction product of nitric oxide (NO) and superoxide, peroxynitrite, can induce mitochondrial biogenesis through Peroxisome proliferator-activated receptor gamma coactivator 1 -alpha $(\mathrm{PGC} 1 \alpha)$ and this, in turn, is modulated by the levels and activity of mitochondrial superoxide dismutase (SOD). ${ }^{[50]}$ Since mitochondria are a potentially controlled site of superoxide production and a target for NO-dependent modulation of respiration, this provides a novel and interesting mechanism for maintenance of mitochondrial quality by stimulating the synthesis of new organelles under conditions of oxidative stress. ${ }^{[1,52]}$ The post-translational modification of proteins mediated by NO remains an area of intense interest, particularly in the mitochondrion. ${ }^{[53]}$ The mitochondrion is potentially particularly susceptible since it contains high concentrations of reactive protein thiols and has long been thought to be a major site for $S$-nitrosation. ${ }^{[41,54-56]}$ Interestingly, under conditions of oxidative stress, degradation of mitochondrial $S$-nitrosothiols appears to be inhibited and this enhances toxicity in the endothelial cells. ${ }^{[57]}$

From a therapeutic perspective, the need to control mitochondrial ROS and the associated signaling has resulted 
in several different strategies to modulate mitochondrial ROS generation both with small molecules and using molecular biology approaches..$^{[12,13,15,58-60]}$ The matrix of the mitochondrion is highly negatively charged and this has been exploited in a series of compounds using a delocalized cationic charge to transport functional pharmacophores into the mitochondrion. The best understood of these compounds is mitochondrially targeted ubiquinone or MitoQ, which is well tolerated in human subjects. ${ }^{[61]}$ It seems likely that the most appropriate therapeutic application of these compounds relates to the signaling pathways modulated by MitoQ which are deranged under conditions of metabolic stress including diabetes, metabolic syndrome, and alcohol-dependent hepatotoxicity. ${ }^{[2-64]}$ The best understood of these targeting molecules is the triphenylphosphonium (TPP+) group which can be coupled to a broad range of pharmacophores and redox-sensitive probes. ${ }^{[16,65,66]}$ These molecules have been used both in vivo and in cell culture to probe the mechanisms of redox signaling, and the effects they elicit are generally ascribed to the properties of the functional group and not the carrying molecule. However, it was recently shown that the TPP + group itself can modulate mitochondrial bioenergetics and the effects vary with the linker group used to attach the functional pharmacophore. ${ }^{[67]}$ The concentrations needed to have these effects cannot usually be achieved in vivo, but in cell culture, where treatment conditions are not usually constrained by pharmacokinetics, the effects of the carrier group need to be taken into account. Some of the effects ascribed to mitochondrially targeted antioxidants may, in fact, be due to off-target effects such as uncoupling, rather than ROS scavenging.

The adenoviral expression of therapeutic proteins has long been recognized as an important approach to redox therapeutics. Overexpression of the cytosolic isoform of SOD in neurons results in its localization to the mitochondrion, where it is capable of modulating mitochondrial superoxide generation in response to angiotensin II. ${ }^{[68]}$ Importantly, the functional impact of modulating mitochondrial superoxide/ hydrogen peroxide is to prevent the angiotensin II-induced neuronal potassium current. Angiotensin II is well known to induce superoxide formation through activation of NADPH oxidase, but it is also becoming clear that mitochondrial superoxide is playing a role in its induction of senescence in vascular smooth muscle cells. ${ }^{[69]}$

Although the mitochondrion has a complex integrated redox modulatory system, there is a selective pressure against the incorporation the antioxidant enzyme, heme oxygenase into the organelle. In support of this idea, mitochondria-targeted heme oxygenase- 1 has recently been reported to suppress the levels of mitochondrial heme proteins and cause oxidative stress and mitochondrial dysfunction in macrophages and other cell types. ${ }^{[70,71]}$

\section{Mitochondrial quality control and novel modulators of mitochondrial function}

The mitochondrial population is in a dynamic equilibrium with a balance maintained between biogenesis and mitophagy. ${ }^{[1,2,72-74]}$ Interestingly, it now appears that polyphenolics may be capable of activating autophagy and may exert their protective effects through this mechanism. ${ }^{[74,75]}$ One of the most important pathways in the cell promoting oxidative stress is the activation of the cytochrome P450 enzymes which are capable of metabolizing a broad range of xenobiotics to form ROS. It is now becoming clear that in addition to the suppression of toxic xenobiotic reactive species by intracellular antioxidants, damaged proteins and organelles must also be removed by the autophagic/lysosomal system. ${ }^{[76,77]}$ One of the commonest causes of liver failure in human subjects is overdose with acetaminophen. Investigation of the mechanisms of toxicity of this compound have revealed that mitochondria are a primary target, and have also revealed a novel mediator of bioenergetic dysfunction, the mitochondrial spheroids. ${ }^{[78]}$ The critical importance of autophagy in bioenergetic dysfunction was further demonstrated in an interesting study in which inhibition of autophagy during chronic alcohol exposure and by several other xenobiotics increased hepatotoxicity, but this was prevented in a cytochrome P450 knockout model. ${ }^{[79]}$ An important alternative mechanism for the removal of oxidized proteins in the mitochondria is their proteolytic degradation mediated by the Lon protease. Under conditions of acute stress, Lon is rapidly induced; but under conditions of chronic stress, aging, and senescence, the levels decline leaving the cell particularly vulnerable to bioenergetic dysfunction. ${ }^{[80]}$

The oxygen binding site in the mitochondrion at cytochrome $c$ oxidase is well known to be the site of interaction with NO. ${ }^{[53]}$ Since the synthesis of NO by nitric oxide synthases also requires oxygen, this establishes an interesting and potentially important biological interaction between oxygen and NO gradients in organs and tissues. ${ }^{[1,53]}$ Interestingly, under conditions of very low oxygen tension or hypoxia, nitrite, a metabolite of NO, can be converted by heme proteins such as myoglobin back to NO, and this can then modulate mitochondrial function. ${ }^{[81]}$

Hydrogen sulfide $\left(\mathrm{H}_{2} \mathrm{~S}\right)$ is the latest member of the gaseous molecules found to be capable of being generated within cells through metabolic processes. Interestingly, $\mathrm{H}_{2} \mathrm{~S}$ has the potential to modulate respiration both at cytochrome $c$ oxidase and through modulation of the thiol redox state in the mitochondrion. ${ }^{[82]}$ This is clearly an area which is likely to develop rapidly given the emerging 
importance of thiol networks in regulating mitochondrial function. ${ }^{[56]}$

Post-translational modifications that affect the mitochondrial function include phosphorylation and protein acetylation. ${ }^{[83,84]}$ Acetylation of lysines within complex I, II, and $\mathrm{V}$ of the electron transport chain and enzymes that control fatty acid oxidation, glycolysis, and amino acid metabolism was shown to reduce the enzyme activity and decrease ATP production. Most often recognized for their role in increasing longevity upon caloric restriction, sirtuins have additionally been shown to control the stress response and, most recently, to mediate the metabolism through direct deacetylation of metabolic enzymes. Mitochondrial Sirt3, specifically, has been shown to sense and control the metabolism through its enzymatic action which is NAD + dependent, and to increase the activity of antioxidant enzymes such as manganese superoxide dismutase (MnSOD) ${ }^{[84]}$ Dysregulation of Sirt3 has pleiotropic effects and is thought to be associated with metabolic syndrome, and other metabolic pathologies including aging, high fat diet, oxidative stress, and ethanol consumption. ${ }^{[84]}$

\section{Translational bioenergetics}

Mitochondrial dysfunction is associated with a broad range of metabolic pathologies including diabetes, neurodegeneration, and cardiovascular diseases ${ }^{[85]}$ Although the clinical focus with many of these diseases is based upon specific abnormalities in the organ function which impact the patient's health, the effects are systemic and reflected in many tissues including circulating platelets and leukocytes. ${ }^{[86,87]}$ Recognition of this concept has led to the hypothesis that these cells can act as sensors or biomarkers of these pathologies. Testing this hypothesis over the last 20-30 years, most frequently with platelets, has resulted in a robust literature showing that bioenergetics defects can be detected in these cells from patients with diabetes or neurodegenerative disease. ${ }^{[86]}$ The recent development of high-throughput methods to isolate specific cell populations from small quantities of patient's blood and assess their bioenergetics is providing the impetus for the next phase of this field. It is now becoming possible to define a patient's "bioenergetics health" which may be of both prognostic and diagnostic value. Using a mitochondrial stress test, it is now possible to determine bioenergetic health index (BHI) which is a single value that can define the bioenergetic health in the cells isolated from a patient's blood. ${ }^{[88]}$ Importantly, a comparison of the bioenergetics and glycolysis of leukocytes and platelets from the patient conclusively demonstrates that their metabolism is distinct and they can, therefore, act as differential sensors of metabolic defects in human subjects. ${ }^{[87,89]}$
Interestingly, a mitochondrial involvement in pathologies not directly related to the organelle is beginning to emerge. An interesting example is cystic fibrosis in which it appears that the defects in the cystic fibrosis transmembrane conductance regulator protein CFTR result in perturbations in cellular bioenergetics including suppressed complex I activity. ${ }^{[90,91]}$

\section{Conclusion}

The field of bioenergetics is now rapidly developing to encompass all aspects of redox biology, as summarized in Figure 1. This includes defining how the organelle regulates cell signaling under physiological conditions and how the cells of the innate immune system change their metabolism in response to their evolving role in inflammation. We are now also on the threshold of the emergence of the new field of translational bioenergetics and the applications of mitochondrial therapeutics.

\section{Acknowledgements}

The authors appreciate support from the NIH T32 training grant T32HL07918 (PAK), the NIDDK Diabetic

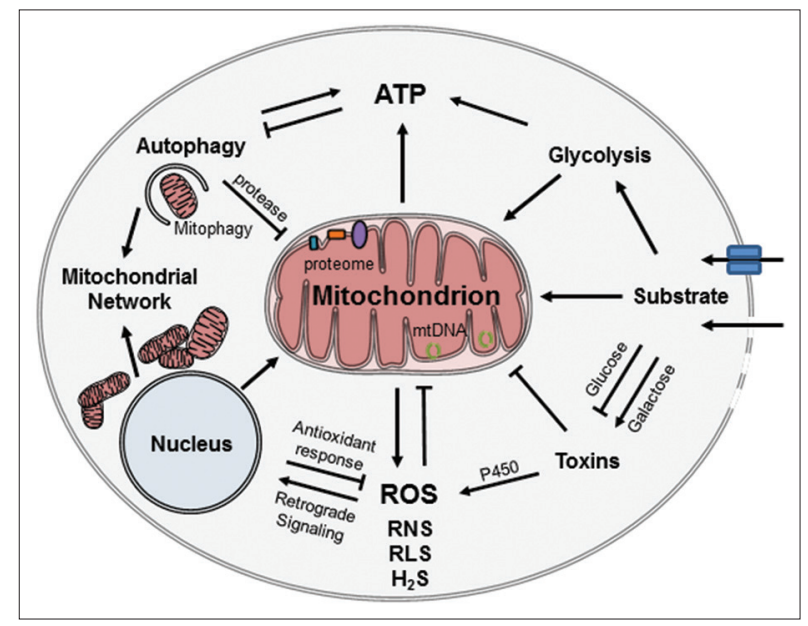

Figure 1: Bioenergetics and its interface with redox biology. Mitochondria are known to generate ATP and reactive oxygen species (ROS) as independently regulated products of metabolism. Critically, substrate availability, toxins, nuclear crosstalk, and mitochondrial biogenesis and degradation all play a role in mitochondrial efficiency and the redox environment. Mitochondrial components such as DNA (mtDNA) and the mitochondrial proteome can be affected by processes such as protease activity, ROS-mediated DNA damage, and mitochondrial dynamics (fission and fusion). Damage resulting to this highly energetic and redox-sensitive organelle can result in an increase in autophagic removal of the mitochondria (mitophagy) and disruption of the mitochondrial network. Other redox active molecules such as reactive nitrogen, lipid species, and hydrogen sulfide have been implicated in oxidative stress and mitochondrial damage. Monitoring mitochondrial function in translational studies can provide insight into the complex interaction between redox biology and cellular bioenergetics. 
Complications Consortium (DiaComp, http://www.diacomp. org) Grant DK076169 (sub-award VDU), and the O'Brien Center P30 DK079337.

\section{REFERENCES}

1. Zhang J. Autophagy and Mitophagy in Cellular Damage Control. Redox Biol 2013;1:19-23.

2. Hill BG, Benavides GA, Lancaster JR Jr, Ballinger S, Dell'Italia L, Jianhua Z, et al. Integration of cellular bioenergetics with mitochondrial quality control and autophagy. Biol Chem 2012;393:1485-512.

3. Lemasters JJ. Selective mitochondrial autophagy, or mitophagy, as a targeted defense against oxidative stress, mitochondrial dysfunction, and aging. Rejuvenation Res 2005;8:3-5.

4. Bereiter-Hahn J, Jendrach M. Mitochondrial dynamics. Int Rev Cell Mol Biol 2010;284:1-65.

5. Narendra DP, Youle RJ. Neurodegeneration: Trouble in the cell's powerhouse. Nature 2012;483:418-9.

6. Santos RX, Correia SC, Carvalho C, Cardoso S, Santos MS, Moreira PI. Mitophagy in neurodegeneration: An opportunity for therapy? Curr Drug Targets 2011;12:790-9.

7. Wallace DC, Fan W, Procaccio V. Mitochondrial energetics and therapeutics. Annu Rev Pathol 2010;5:297-348.

8. Krzywanski DM, Moellering DR, Fetterman JL, Dunham-Snary KJ, Sammy MJ, Ballinger SW. The mitochondrial paradigm for cardiovascular disease susceptibility and cellular function: A complementary concept to Mendelian genetics. Lab Invest 2011;91:1122-35.

9. Harrison CM, Pompilius M, Pinkerton KE, Ballinger SW. Mitochondrial oxidative stress significantly influences atherogenic risk and cytokine-induced oxidant production. Environ Health Perspect 2011;119:676-81.

10. Gutierrez J, Ballinger SW, Darley-Usmar VM, Landar A. Free radicals, mitochondria, and oxidized lipids: The emerging role in signal transduction in vascular cells. Circ Res 2006;99:924-32.

11. Mitchell T, Johnson MS, Ouyang X, Chacko BK, Mitra K, Lei X, et al. Dysfunctional mitochondrial bioenergetics and oxidative stress in Akita(+/Ins2)-derived beta-cells. Am J Physiol Endocrinol Metab 2013;305:E585-99.

12. Horton KL, Stewart KM, Fonseca SB, Guo Q, Kelley SO. Mitochondria-penetrating peptides. Chem Biol 2008;15:375-82.

13. Rehman H, Ramshesh VK, Theruvath TP, Kim I, Currin RT, Giri S, et al. NIM811 (N-methyl-4-isoleucine cyclosporine), a mitochondrial permeability transition inhibitor, attenuates cholestatic liver injury but not fibrosis in mice. J Pharmacol Exp Ther 2008;327:699-706.

14. Burchell VS, Gandhi S, Deas E, Wood NW, Abramov AY, Plun-Favreau H. Targeting mitochondrial dysfunction in neurodegenerative disease: Part II. Expert Opin Ther Targets 2010;14:497-511.

15. Carreira RS, Lee P, Gottlieb RA. Mitochondrial therapeutics for cardioprotection. Curr Pharm Des 2011;17:2017-35.

16. Murphy MP. Targeting lipophilic cations to mitochondria. Biochim Biophys Acta 2008;1777:1028-31.

17. Whitaker-Menezes D, Martinez-Outschoorn UE, Flomenberg N,
Birbe RC, Witkiewicz AK, Howell A, et al. Hyperactivation of oxidative mitochondrial metabolism in epithelial cancer cells in situ: Visualizing the therapeutic effects of metformin in tumor tissue. Cell Cycle 2011;10:4047-64.

18. Dodson M, Darley-Usmar V, Zhang J. Cellular metabolic and autophagic pathways: Traffic control by redox signaling. Free Radic Biol Med 2013;63:207-21.

19. Butow RA, Avadhani NG. Mitochondrial signaling: The retrograde response. Mol Cell 2004;14:1-15.

20. Cadenas E. Mitochondrial free radical production and cell signaling. Mol Aspects Med 2004;25:17-26.

21. Brookes PS, Levonen AL, Shiva S, Sarti P, Darley-Usmar VM. Mitochondria: Regulators of signal transduction by reactive oxygen and nitrogen species. Free Radic Biol Med 2002;33:755-64.

22. Higdon A, Diers AR, Oh JY, Landar A, Darley-Usmar VM. Cell signalling by reactive lipid species: New concepts and molecular mechanisms. Biochem J 2012;442:453-64.

23. Fetterman JL, Zelickson BR, Johnson LW, Moellering DR, Westbrook DG, Pompilius M, et al. Mitochondrial genetic background modulates bioenergetics and susceptibility to acute cardiac volume overload. Biochem J 2013;455:157-67.

24. Pearce EL, Poffenberger MC, Chang CH, Jones RG. Fueling immunity: Insights into metabolism and lymphocyte function. Science 2013;342:1242454.

25. Pearce EL, Pearce EJ. Metabolic pathways in immune cell activation and quiescence. Immunity 2013;38:633-43.

26. Tavakoli S, Zamora D, Ullevig S, Asmis R. Bioenergetic Profiles Diverge During Macrophage Polarization: Implications for the Interpretation of 18F-FDG PET Imaging of Atherosclerosis. J Nucl Med 2013;54:1661-7.

27. Ravi S, Mitchell T, Kramer PA, Chacko B, Darley-Usmar VM. Mitochondria in monocytes and macrophages-implications for translational and basic research. Int J Biochem Cell Biol 2014;53C: 202-7.

28. Block K, Gorin Y, Abboud HE. Subcellular localization of Nox4 and regulation in diabetes. Proc Natal Acad Sci USA 2009;106:14385-90.

29. Bansal S, Anandatheerthavarada HK, Prabu GK, Milne GL, Martin MV, Guengerich FP, et al. Human cytochrome P450 2E1 mutations that alter mitochondrial targeting efficiency and susceptibility to ethanol-induced toxicity in cellular models. J Biol Chem 2013;288:12627-44.

30. Finocchietto PV, Franco MC, Holod S, Gonzalez AS, Converso DP, Arciuch VG, et al. Mitochondrial nitric oxide synthase: A masterpiece of metabolic adaptation, cell growth, transformation, and death. Exp Biol Med (Maywood) 2009;234:1020-8.

31. Salabei JK, Gibb AA, Hill BG. Comprehensive measurement of respiratory activity in permeabilized cells using extracellular flux analysis. Nat Protoc 2014;9:421-38.

32. Salabei JK, Hill BG. Mitochondrial fission induced by platelet-derived growth factor regulates vascular smooth muscle cell bioenergetics and cell proliferation. Redox Biol 2013;1:542-51.

33. Diers AR, Vayalil PK, Oliva CR, Griguer CE, Darley-Usmar V, Hurst DR, et al. Mitochondrial bioenergetics of metastatic breast cancer cells in response to dynamic changes in oxygen tension: Effects of HIF-1alpha. PloS One 2013;8:e68348.

34. Xu W, Janocha AJ, Leahy RA, Klatte R, Dudzinski D, Mavrakis LA, 
et al. A novel method for pulmonary research: Assessment of bioenergetic function at the air-liquid interface. Redox Biol 2014;2:513-9.

35. Benavides GA, Liang Q, Dodson M, Darley-Usmar V, Zhang J. Inhibition of autophagy and glycolysis by nitric oxide during hypoxia-reoxygenation impairs cellular bioenergetics and promotes cell death in primary neurons. Free Radic Biol Med 2013;65:1215-28.

36. Zelickson BR, Benavides GA, Johnson MS, Chacko BK, Venkatraman A, Landar A, et al. Nitric oxide and hypoxia exacerbate alcohol-induced mitochondrial dysfunction in hepatocytes. Biochim Biophys Acta 2011;1807:1573-82.

37. Dier U, Shin DH, Hemachandra LP, Uusitalo LM, Hempel N. Bioenergetic analysis of ovarian cancer cell lines: Profiling of histological subtypes and identification of a mitochondria-defective cell line. PloS One 2014;9:e98479.

38. Misztal T, Rusak T, Tomasiak M. Clinically relevant $\mathrm{HOCl}$ concentrations reduce clot retraction rate via the inhibition of energy production in platelet mitochondria. Free Radic Res 2014;48:1443-53.

39. Gohil VM, Sheth SA, Nilsson R, Wojtovich AP, Lee JH, Perocchi F, et al. Nutrient-sensitized screening for drugs that shift energy metabolism from mitochondrial respiration to glycolysis. Nature biotechnology 2010;28:249-55.

40. Dott W, Mistry P, Wright J, Cain K, Herbert KE. Modulation of mitochondrial bioenergetics in a skeletal muscle cell line model of mitochondrial toxicity. Redox Biol 2014;2:224-33.

41. Murphy M. Mitochondrial Thiols in Antioxidant Protection and Redox Signaling: Distinct roles for glutathionylation and other thiol modifications. Antioxid Redox Signal 2011;16:476-495.

42. Murphy MP. How mitochondria produce reactive oxygen species. Biochem J 2009;417:1-13.

43. Jazwinski SM. The retrograde response: When mitochondrial quality control is not enough. Biochim Biophys Acta 2013;1833:400-9.

44. Scandroglio F, Tortora V, Radi R, Castro L. Metabolic control analysis of mitochondrial aconitase: Influence over respiration and mitochondrial superoxide and hydrogen peroxide production. Free Radic Res 2014;48:684-93.

45. Boveris A, Chance B. The mitochondrial generation of hydrogen peroxide. General properties and effect of hyperbaric oxygen. Biochem J 1973;134:707-16.

46. Quinlan CL, Perevoshchikova IV, Hey-Mogensen M, Orr AL, Brand MD. Sites of reactive oxygen species generation by mitochondria oxidizing different substrates. Redox Biol 2013;1:304-12.

47. Watanabe N, Zmijewski JW, Takabe W, Umezu-Goto M, Le Goffe C, Sekine A, et al. Activation of mitogen-activated protein kinases by lysophosphatidylcholine-induced mitochondrial reactive oxygen species generation in endothelial cells. Am J Pathol 2006; $168: 1737-48$.

48. Trujillo J, Chirino YI, Molina-Jijon E, Anderica-Romero AC, Tapia E, Pedraza-Chaverri J. Renoprotective effect of the antioxidant curcumin: Recent findings. Redox Biol 2013;1:448-56.

49. Koenitzer JR, Freeman BA. Redox signaling in inflammation: Interactions of endogenous electrophiles and mitochondria in cardiovascular disease. Ann N Y Acad Sci 2010;1203:45-52.

50. Marine A, Krager KJ, Aykin-Burns N, Macmillan-Crow LA.
Peroxynitrite induced mitochondrial biogenesis following MnSOD knockdown in normal rat kidney (NRK) cells. Redox Biol 2014;2:348-57.

51. Shiva S, Oh JY, Landar AL, Ulasova E, Venkatraman A, Bailey SM, et al. Nitroxia: The pathological consequence of dysfunction in the nitric oxide-cytochrome c oxidase signaling pathway. Free Radic Biol Med 2005;38:297-306.

52. Erusalimsky JD, Moncada S. Nitric oxide and mitochondrial signaling: From physiology to pathophysiology. Arterioscler Thromb Vasc Biol 2007;27:2524-31.

53. Hill BG, Dranka BP, Bailey SM, Lancaster JR Jr, Darley-Usmar VM. What part of NO don't you understand? Some answers to the cardinal questions in nitric oxide biology. J Biol Chem 2010;285:19699-704.

54. Chouchani ET, Hurd TR, Nadtochiy SM, Brookes PS, Fearnley IM, Lilley KS, et al. Identification of S-nitrosated mitochondrial proteins by S-nitrosothiol difference in gel electrophoresis (SNO-DIGE): Implications for the regulation of mitochondrial function by reversible S-nitrosation. Biochem J 2010;430:49-59.

55. Burwell LS, Nadtochiy SM, Tompkins AJ, Young S, Brookes PS. Direct evidence for S-nitrosation of mitochondrial complex I. Biochem J 2006;394:627-34.

56. Mailloux RJ, Jin X, Willmore WG. Redox regulation of mitochondrial function with emphasis on cysteine oxidation reactions. Redox Biol 2013;2:123-39.

57. Diers AR, Broniowska KA, Hogg N. Nitrosative stress and redox-cycling agents synergize to cause mitochondrial dysfunction and cell death in endothelial cells. Redox Biol 2013;1:1-7.

58. Murphy MP. Development of lipophilic cations as therapies for disorders due to mitochondrial dysfunction. Expert Opin Biol Ther 2001;1:753-64

59. Muratovska A, Lightowlers RN, Taylor RW, Wilce JA, Murphy MP. Targeting large molecules to mitochondria. Adv Drug Deliv Rev 2001;49:189-98

60. Prime TA, Blaikie FH, Evans C, Nadtochiy SM, James AM, Dahm CC, et al. A mitochondria-targeted S-nitrosothiol modulates respiration, nitrosates thiols, and protects against ischemia-reperfusion injury. Proc Natl Acad Sci USA 2009;106:10764-9.

61. Smith RA, Murphy MP. Animal and human studies with the mitochondria-targeted antioxidant MitoQ. Ann N Y Acad Sci 2010;1201:96-103.

62. Mercer JR, Yu E, Figg N, Cheng KK, Prime TA, Griffin JL, et al. The mitochondria-targeted antioxidant MitoQ decreases features of the metabolic syndrome in ATM+/-/ApoE-/- mice. Free Radic Biol Med 2012;52:841-9.

63. Chacko BK, Srivastava A, Johnson MS, Benavides GA, Chang MJ, Ye Y, et al. Mitochondria-targeted ubiquinone (MitoQ) decreases ethanol-dependent micro and macro hepatosteatosis. Hepatology 2011;54:153-63.

64. Chacko BK, Reily C, Srivastava A, Johnson MS, Ye YZ, Ulasova E, et al. Prevention of diabetic nephropathy in Ins2(+/-AkitaJ) mice by the mitochondria-targeted therapy MitoQ. Biochem J 2010;432:9-19.

65. Millard M, Pathania D, Shabaik Y, Taheri L, Deng J, Neamati N. Preclinical evaluation of novel triphenylphosphonium salts with broad-spectrum activity. PloS One 2010;5:e13131.

66. Pun PB, Logan A, Darley-Usmar V, Chacko B, Johnson MS, Huang $\mathrm{GW}$, et al. A mitochondria-targeted mass spectrometry probe 
to detect glyoxals: Implications for diabetes. Free Radic Biol Med 2014;67:437-50.

67. Reily C, Mitchell T, Chacko BK, Benavides G, Murphy MP, Darley-Usmar V. Mitochondrially targeted compounds and their impact on cellular bioenergetics. Redox Biol 2013;1:86-93.

68. Li S, Case AJ, Yang RF, Schultz HD, Zimmerman MC. Over-expressed copper/zinc superoxide dismutase localizes to mitochondria in neurons inhibiting the angiotensin II-mediated increase in mitochondrial superoxide. Redox Biol 2013;2:8-14.

69. Mistry Y, Poolman T, Williams B, Herbert KE. A role for mitochondrial oxidants in stress-induced premature senescence of human vascular smooth muscle cells. Redox Biol 2013;1:411-7.

70. Bolisetty S, Traylor A, Zarjou A, Johnson MS, Benavides GA, Ricart K, et al. Mitochondria-targeted heme oxygenase-1 decreases oxidative stress in renal epithelial cells. Am J Physiol Renal Physiol 2013;305:F255-64.

71. Bansal S, Biswas G, Avadhani NG. Mitochondria-targeted heme oxygenase-1 induces oxidative stress and mitochondrial dysfunction in macrophages, kidney fibroblasts and in chronic alcohol hepatotoxicity. Redox Biol 2014;2:273-83.

72. Kim I, Rodriguez-Enriquez S, Lemasters JJ. Selective degradation of mitochondria by mitophagy. Arch Biochem Biophys 2007;462:245-53.

73. Giordano S, Darley-Usmar V, Zhang J. Autophagy as an essential cellular antioxidant pathway in neurodegenerative disease. Redox Biol 2014;2:82-90.

74. Liu J, Tang Y, Feng Z, Liu J, Liu J, Long J.(-)-Epigallocatechin-3-gallate attenuated myocardial mitochondrial dysfunction and autophagy in diabetic Goto-Kakizaki rats. Free Radic Res 2014;48:898-906.

75. Kim HS, Quon MJ, Kim JA. New insights into the mechanisms of polyphenols beyond antioxidant properties; lessons from the green tea polyphenol, epigallocatechin 3-gallate. Redox Biol 2014;2:187-95.

76. Lee J, Giordano S, Zhang J. Autophagy, mitochondria and oxidative stress: Cross-talk and redox signalling. Biochem J 2012;441:523-40.

77. Haberzettl P, Hill BG. Oxidized lipids activate autophagy in a JNK-dependent manner by stimulating the endoplasmic reticulum stress response. Redox Biol 2013;1:56-64.

78. Ni HM, Williams JA, Jaeschke H, Ding WX. Zonated induction of autophagy and mitochondrial spheroids limits acetaminophen-induced necrosis in the liver. Redox Biol 2013;1:427-32.

79. Wu D, Cederbaum AI. Inhibition of autophagy promotes
CYP2E1-dependent toxicity in HepG2 cells via elevated oxidative stress, mitochondria dysfunction and activation of p38 and JNK MAPK. Redox Biol 2013;1:552-65.

80. Ngo JK, Pomatto LC, Davies KJ. Upregulation of the mitochondrial Lon Protease allows adaptation to acute oxidative stress but dysregulation is associated with chronic stress, disease, and aging. Redox Biol 2013;1:258-64.

81. Shiva S. Nitrite: A Physiological Store of Nitric Oxide and Modulator of Mitochondrial Function. Redox Biol 2013;1:40-4.

82. Stein A, Bailey SM. Redox Biology of Hydrogen Sulfide: Implications for Physiology, Pathophysiology, and Pharmacology. Redox Biol 2013;1:32-9.

83. Foster DB, Van Eyk JE, Marban E, O'Rourke B. Redox signaling and protein phosphorylation in mitochondria: Progress and prospects. J Bioenerg Biomembr 2009;41:159-68.

84. Wu YT, Wu SB, Wei YH. Roles of sirtuins in the regulation of antioxidant defense and bioenergetic function of mitochondria under oxidative stress. Free Radic Res 2014;48:1070-84.

85. Areti A, Yerra VG, Naidu V, Kumar A. Oxidative stress and nerve damage: Role in chemotherapy induced peripheral neuropathy. Redox Biol 2014;2:289-95.

86. Zharikov S, Shiva S. Platelet mitochondrial function: From regulation of thrombosis to biomarker of disease. Biochem Soc Trans 2013;41:118-23.

87. Chacko BK, Kramer PA, Ravi S, Johnson MS, Hardy RW, Ballinger SW, et al. Methods for defining distinct bioenergetic profiles in platelets, lymphocytes, monocytes, and neutrophils, and the oxidative burst from human blood. Lab Invest 2013;93:690-700.

88. Chacko BK, Kramer PA, Ravi S, Benavides GA, Mitchell T, Dranka BP, et al. The Bioenergetic Health Index: A new concept in mitochondrial translational research. Clin Sci (Lond) 2014;127:367-73.

89. Kramer PA, Ravi S, Chacko B, Johnson MS, Darley-Usmar VM A review of the mitochondrial and glycolytic metabolism in human platelets and leukocytes: Implications for their use as bioenergetic biomarkers. Redox Biol 2014;2:206-10.

90. Valdivieso AG, Santa-Coloma TA. CFTR activity and mitochondrial function. Redox Biol 2013;1:190-202.

91. Valdivieso AG, Clauzure M, Marin MC, Taminelli GL, Massip Copiz MM, Sanchez F, et al. The mitochondrial complex I activity is reduced in cells with impaired cystic fibrosis transmembrane conductance regulator (CFTR) function. PloS One 2012;7:e48059. 